\title{
INSATISFAÇÃO COM A IMAGEM CORPORAL EM ADOLESCENTES E SUA RELAÇÃO COM A MATURAÇÃO SEXUAL
}

\author{
BODY IMAGE DISSATISFACTION DURING ADOLESCENCE AND ITS RELA- \\ TION TO SEXUAL MATURATION
}

\author{
Maria A Conti ${ }^{*}$ \\ Ana M D Gambardella** \\ Maria F P Frutuoso ${ }^{* * *}$
}

\begin{abstract}
Conti MA, Gambardella AMD, Frutuoso MFP. Insatisfação com a imagem corporal em adolescentes e sua relação com a maturação sexual. Rev Bras Cresc Desenv Hum 2005; 15(2):36-44.

Resumo: Sérios problemas comportamentais envolvendo hábitos alimentares, com repercussão na percepção da imagem corporal, tornam-se cada vez mais comuns. O presente estudo objetivou avaliar a percepção da insatisfação da imagem corporal de um grupo de adolescentes. Pesquisouse jovens de uma Instituição de Rede Particular de Ensino Fundamental, de 10 a 14 anos, de ambos os sexos. Para análise de dados utilizou-se como critério a idade, transformada em fase de maturação sexual. Para avaliar a percepção da imagem corporal aplicou-se escala de satisfação adaptada, composta por 15 áreas corporais. Registrou-se para meninos, maior insatisfação para peso corporal, cintura e estômago e meninas para peso corporal, tórax/seio e estômago. Na associação entre insatisfação corporal e maturação corporal, as áreas significantes foram, para os meninos, ombro/costas e para as meninas, rosto, cabelo, quadril, estômago, cintura, tórax/seio, tônus muscular, altura e aspectos gerais. Conclui-se que meninas pós-púberes mostraram-se mais suscetíveis à manifestação da insatisfação corporal quando comparadas aos seus pares.
\end{abstract}

Palavras-chave: Adolescência. Imagem corporal. Maturação sexual.

\section{INTRODUÇÃO}

A adolescência pode ser compreendida como uma etapa evolutiva peculiar do ser humano. Nela culmina o processo maturativo biopsicossocial do indivíduo, motivo pelo qual não se pode compreender a adolescência estudando separadamente os aspectos biológicos, psicológicos, sociais ou culturais. Tais aspectos, indissociáveis, constituem justamente o conjunto de características que confere a unidade do fenômeno da adolescência ${ }^{1}$.

As teorias relacionadas à adolescência priorizam o aspecto cronológico quando conceituam-na com base na faixa etária ou ao aspecto físico, ao associá-la ao crescimento físico e maturação puberal ${ }^{2}$; ou ao aspecto psicológico em função da reorganização da identidade profissional, sexual e filosófica ${ }^{3}$; ou ao enfocar o aspecto sociológico, no sentido da necessidade do reencontro do papel social deste individuo na sociedade ${ }^{4}$.

Da dissertação de Mestrado: "Imagem corporal e estado nutricional de estudantes de uma escola da rede particular", Departamento de Nutrição FSP/USP, 2002. Doutoranda em Saúde Pública pelo Departamento de Nutrição da Facul dade de Saúde Pública da USP . Rua Arthur Corradi,, 120 - 3º andar - CEP: 09725-240 - Vila Duzzi, São Bernardo do Campo-SP. Fone: (011) 4125-8619/4232-2345 - e-mail: maconti@usp.br.

** Professora Doutora - Departamento de Nutrição da Faculdade de Saúde Pública da USP.

*** Professora Doutora - Departamento de Nutrição da Faculdade de Saúde Pública da USP. 
Contudo, sabe-se que a adolescência tem características específicas de acordo com o nível socioeconômico no qual este jovem está inserido e que acaba por determinar formas diferentes de ser adolescente ${ }^{5}$. O jovem dos dias atuais, com dedicação exclusiva ao estudo, com períodos livres para lazer e socialização, viverá uma adolescência diferente daquele que concilia a obrigação de uma atividade produtiva remunerada ao estudo, ou até daquele que abdica da escola. De acordo com Salles ${ }^{5}$, a inserção social deste jovem define o modo de ser adolescente, assim como sua conduta, aspirações e responsabilidades. Vaccari ${ }^{6}$ complementa afirmando haver mais do que uma adolescência, no singular, sugerindo adolescências, no plural, considerando-se as diferenças de gênero, classe social, região do país, etc.

No campo da Saúde, o critério cronológico é usado correntemente, entendendo adolescentes como indivíduos de 10 a 19 anos de idade $^{2}$, sendo o desenvolvimento físico um dos fatores de grande influência para esta etapa do desenvolvimento humano. Biologicamente, é a fase de maior velocidade de crescimento ${ }^{7}$. Ser adolescente, dos 10 aos 14 anos representa uma fase de grandes mudanças físicas e corporais o que implica em alterações na aparência física e tamanho corporal, afetando diretamente a imagem e satisfação corporais ${ }^{8}$.

Os adolescentes, com as mudanças biológicas e maturação sexual, devem incorporar suas novas imagens corporais, capacidade reprodutiva e energia sexual emergente para sua identidade, bem como aprender a enfrentar a sua própria reação e a dos demais à maturação corporal. Há diferenças biológicas básicas para meninos e meninas no tempo pubertário e na construção social do gênero em relação ao significado e ação do adolescente ${ }^{9}$.

Estudos utilizando fase da adolescência, ou tempo relativo de desenvolvimento, indicam que este tempo influencia sentimentos sobre puberdade, satisfação e imagem corporais, en- tre outros comportamentos psicológicos em meninos e meninas ${ }^{10}$.

No entanto, há décadas, meninas recebem maior atenção investigativa, constatando insatisfação corporal ${ }^{11,17}$. Estudos recentes inferem esta mesma preocupação envolvendo o gênero masculino ${ }^{8,9}$. Pesquisas sobre gêneros são recentes, trazendo à tona questões como, por exemplo, a diferença entre meninos e meninas no que tange à imagem corporal e aos possíveis fatores que possam influenciar estes jovens.

Sabe-se que, entre mulheres da cultura ocidental, preocupações com a imagem e o peso corporal são comuns. Estudos populacionais têm documentado que a maioria das mulheres, adolescentes ou jovens, mostra-se insatisfeita com a imagem corporal, embora só a minoria esteja realmente com sobrepeso ${ }^{14}$.

Sérios problemas comportamentais, envolvendo hábitos alimentares, podem repercutir negativamente na percepção da imagem corporal, especialmente entre jovens. De um pólo a outro, da obesidade à anorexia, jovens desenvolvem distúrbios de conduta alimentar comprometendo sua saúde e qualidade de vida.

A prevalência da obesidade tem aumentado, sendo considerada atualmente como uma epidemia global pela OMS, atingindo crianças, adolescentes e adultos ${ }^{15}$. Dados recentes comprovam o aumento de prevalência de obesidade, em especial entre os adolescentes. Wang et al. ${ }^{16}$ constataram aumento de aproximadamente $9 \%$ na prevalência de obesidade entre adolescentes brasileiros de 6 a 24 anos de idade em um período de 20 anos.

A anorexia e a bulimia nervosa também se constituem em outro problema de comportamento alimentar, denominados transtornos alimentares. Estima-se que de 0,5 a $1 \%$ das adolescentes norte-americanas, entre 12 a 18 anos, podem ser diagnosticadas com anorexia nervosa e de $1 \%$ a $3 \%$ com bulimia ${ }^{17}$.

Independente do sexo, os adolescentes preocupam-se com peso e tamanho corporais 
e aparência. Essa preocupação desmedida pode estar desencadeando hábitos alimentares inadequados e, em decorrência, potencial problema de saúde pública ${ }^{18}$.

É importante ressaltar que a distorção da imagem corporal, super ou sub estimando tamanho e/ou forma do corpo, não se constitui em característica particular de adolescentes que desenvolvem algum tipo de transtorno alimentar; uma vez que se torna cada vez mais presente na dinâmica vivencial dos adolescentes.

Estudo longitudinal, desenvolvido por Archibald et al. ${ }^{17}$, constatou que a insatisfação quanto ao peso e aparência corporais tendem a aumentar durante a adolescência, podendo trazer problemas comportamentais ou ainda ser preditor de transtornos alimentares para alguns jovens.

Pela escassez de estudos nacionais relacionados ao tema e à população pesquisada, $\mathrm{e}$ tendo em vista a importância destes conhecimentos, investigou-se a percepção da insatisfação da imagem corporal em adolescentes.

\section{MÉTODOS}

O estudo foi desenvolvido em uma Instituição da Rede Particular de Ensino Fundamental, localizada no município de Santo André S.P., com adolescentes de 10 a 14 anos de idade, do sexo masculino e feminino. Para a seleção da amostra, adotou-se o critério de amostra casual simples ${ }^{19}$. Foram selecionados 187 alunos (50\% do total) e participaram da pesquisa 147 jovens. Os dados foram obtidos por meio de um questionário respondido pelo próprio adolescente.

\section{Fase da adolescência}

Para avaliar a fase da adolescência, transformou-se idade cronológica em fase de maturação sexual. Deste modo, meninos de 10|-12 anos foram considerados pré-púberes e aqueles de 13|- 14 anos púberes, considerando-se a idade média do surgimento dos pelos axilares (13 anos); meninas de 10l-11 anos foram consideradas púberes e de 12l-14 anos pós-púberes, considerando-se a idade média de ocorrência da menarca $(12 \text { anos })^{2}$.

\section{Insatisfação com a imagem corporal}

A insatisfação com a imagem corporal foi verificada com base na escala de áreas corporais proposta e validada por Brown et al. ${ }^{20} \mathrm{e}$ adaptada por Loland ${ }^{21}$ que consta de 15 áreas corporais, a saber: rosto, cabelo, nádegas, quadril, coxas, pernas, estômago, cintura, seio/tórax, costas/ombros, braços, tônus muscular, peso, altura, todas as áreas.

Solicitou-se a cada adolescente assinalar o seu grau de satisfação em relação a cada área mencionada segundo uma escala tipo likert, variando de muito insatisfeito (1) a muito satisfeito (5). O critério insatisfação foi determinado pela resposta (1) muito insatisfeito e (2) medianamente insatisfeito.

Para análise estatística, além do critério insatisfeito, utilizou-se o critério (3) medianamente satisfeito e (4) satisfeito e (5) muito satisfeito, categorizando-se assim as respostas em 3 níveis: Insatisfeito, medianamente satisfeito e satisfeito.

\section{Análise}

Para verificar as relações de associação entre as variáveis fase de adolescência, para as meninas em púberes e pós- púberes e meninos pré-púberes e púberes e grau de satisfação corporal, com variação entre insatisfeito, medianamente satisfeito e satisfeito, utilizou-se o teste qui-quadrado $\left(\mathrm{c}^{2}\right)$, adotando-se nível de significância de 5\%, com auxílio do programa estatístico Epi-Info - Versão 6.04. 
O projeto foi aprovado pelo Comitê de Ética em Pesquisa de uma Instituição de Ensino Superior Pública e os pais e/ou responsáveis firmaram termo de consentimento para participação da pesquisa.

\section{RESULTADOS}

Participaram do estudo 147 adolescentes, sendo $52(35,3 \%)$ do sexo masculino e 95 $(64,6 \%)$ do sexo feminino. Conforme se observa nas Tabelas 1 e 2, 67,3\% dos meninos encontravam-se na fase pré-púbere e $71,6 \%$ das meninas na pós-púbere.

No geral, as áreas reportadas de maior insatisfação referem-se ao peso corporal com $32 \%$, estômago com $21 \%$ e coxas com $16 \%$. Para o grupo dos meninos, estas áreas se repetem com $35 \%, 25 \%$ e $23 \%$ respectivamente, como áreas de maior insatisfação. Para meninas, peso corporal prevalece como de maior insatisfação (31\%) seguido de tórax/seio (20\%) e estômago (18\%).

\section{Associação entre fase da adolescência e insatisfação corporal}

Para os meninos estudados, apenas uma área surgiu com associação significantemente estatística (ombro/costas), com meninos prépúberes mais insatisfeitos quando comparados

Tabela 1 - Distribuição (\%) dos adolescentes, segundo fase da adolescência, insatisfação e áreas corporais.

\begin{tabular}{lccccccc}
\hline Idade & \multicolumn{3}{c}{$10 \mathrm{l}-12(\mathrm{n}=35)$} & \multicolumn{3}{c}{$\begin{array}{c}\text { 13I-15 }(\mathrm{n}=17) \\
\text { púbere }\end{array}$} \\
\cline { 2 - 7 } Áreas & Insatif & $\begin{array}{c}\text { Med. } \\
\text { Cotisf }\end{array}$ & Satisf & $\begin{array}{c}\text { Insatis } \\
\text { f }\end{array}$ & $\begin{array}{c}\text { Med. } \\
\text { Satisf }\end{array}$ & Satisf & $\chi^{2}(\mathrm{p})$ \\
Corporais & & Satisf & & \\
\hline Rosto & 5,7 & 25,7 & 68,6 & - & 41,2 & 58,8 & $2,03(0,36)$ \\
Cabelo & 11,4 & 28,6 & 60,0 & 11,8 & 11,8 & 76,4 & $1,88(<0,39)$ \\
Nádegas & 14,3 & 37,1 & 48,6 & 23,5 & 41,2 & 35,3 & $1,07(<0,58)$ \\
Quadril & 14,3 & 25,7 & 60,0 & - & 47,1 & 52,9 & $4,12(<0,12)$ \\
Coxas & 20,0 & 22,9 & 57,1 & 29,4 & 23,5 & 47,1 & $0,66(<0,71)$ \\
Pernas & 5,7 & 22,9 & 71,4 & 11,8 & 23,5 & 64,7 & $0,62(<0,73)$ \\
Estômago & 31,4 & 11,4 & 57,2 & 11,8 & 17,6 & 70,6 & $2,43(<0,29)$ \\
Cintura & 25,7 & 22,9 & 51,4 & 17,6 & 23,6 & 58,8 & $0,44(<0,80)$ \\
Tórax/Seio & 8,6 & 17,1 & 74,3 & - & 17,6 & 82,4 & $1,56(<0,45)$ \\
Omb./Cost & 2,9 & 25,7 & 71,4 & - & - & 100,0 & $\mathbf{6 , 0 1}(<\mathbf{0 , 0 4 )})$ \\
Braços & 11,4 & 11,4 & 77,2 & - & 29,4 & 70,6 & $4,15(<0,12)$ \\
T. Musc. & 5,7 & 20,0 & 74,3 & - & 17,6 & 82,4 & $1,10(<0,57)$ \\
Peso & 40,0 & 14,3 & 45,7 & 23,5 & 29,4 & 47,1 & $2,26(<0,32)$ \\
Altura & 8,6 & 22,9 & 68,5 & 17,6 & 17,6 & 64,8 & $0,99(<0,60)$ \\
Geral & 5,7 & 25,7 & 68,6 & - & 17,6 & 82,4 & $1,59(<0,45)$ \\
\hline
\end{tabular}

$\mathrm{p}<0,05$

aos seus pares (Tabela 1).

Para as meninas, rosto, cabelo, quadril, estômago, cintura, tórax/seio, tônus muscular, altura e aspectos gerais apresentaram-se como áreas de significância estatística para associação entre insatisfação corporal e fase de maturação sexual conforme se observa na Tabela 2 . Meninas pós-púberes inferiram maior insatisfação quando comparadas às púberes.

\section{DISCUSSÃO}

Acerca do grupo pesquisado, estes jovens estudavam no período matutino, tendo suas tardes livres para atividades optativas na própria escola como aperfeiçoamento esportivo natação, judô, ginástica rítmica, vôlei; reforço escolar; atividades culturais como teatro ou ainda para encontros com colegas, tendo assim, como 
Tabela 2 - Distribuição (\%) das adolescentes, segundo fase da adolescência, insatisfação e áreas corporais.

\begin{tabular}{|c|c|c|c|c|c|c|c|}
\hline \multirow{2}{*}{$\begin{array}{l}\text { Idade } \\
\text { Áreas Corporais }\end{array}$} & \multicolumn{3}{|c|}{$\begin{array}{c}10 \mid-11(\mathrm{n}=27) \\
\text { púbere }\end{array}$} & \multicolumn{3}{|c|}{$\begin{array}{l}12 \mathrm{l}-15(\mathrm{n}=68) \\
\text { pós-púbere }\end{array}$} & \multirow{2}{*}{$\begin{array}{c}\text { Teste } \\
\chi^{2}(\mathrm{p})\end{array}$} \\
\hline & Insatif & $\begin{array}{l}\text { Med. } \\
\text { Satisf }\end{array}$ & Satisf & Insatisf & $\begin{array}{l}\text { Med. } \\
\text { Satisf }\end{array}$ & Satisf & \\
\hline Rosto & - & 14,8 & 85,2 & 11,8 & 27,9 & 60,3 & $6,33(<0,04)$ \\
\hline Cabelo & - & 11,1 & 88,9 & 11,8 & 35,3 & 52,9 & $11,11(<0,003)$ \\
\hline Nádegas & 3,7 & 14,8 & 81,5 & 13,2 & 23,5 & 63,3 & $3,31(<0,19)$ \\
\hline Quadril & - & 18,5 & 81,5 & 13,2 & 32,4 & 54,4 & $7,16(<0,02)$ \\
\hline Coxas & 11,1 & 14,8 & 74,1 & 11,8 & 30,9 & 57,4 & $2,77(<0,24)$ \\
\hline Pernas & 3,7 & 18,5 & 77,8 & 16,2 & 41,2 & 42,6 & $9,77(<0,07)$ \\
\hline Estômago & 11,1 & 14,8 & 74,1 & 20,6 & 41,2 & 38,2 & $10,08(<0,006)$ \\
\hline Cintura & 3,7 & 14,8 & 81,5 & 16,2 & 27,9 & 55,9 & $5,76(<0,05)$ \\
\hline Tórax/Seio & 3,7 & 18,5 & 77,8 & 26,5 & 29,4 & 44,1 & $9,96(<0,006)$ \\
\hline Omb./Cost & 3,7 & 14,8 & 81,5 & 5,9 & 17,6 & 76,5 & $0,33(<0,84)$ \\
\hline Braços & - & 18,5 & 81,5 & 10,3 & 26,5 & 63,2 & $4,22(<0,12)$ \\
\hline T. Musc. & - & 18,5 & 81,5 & 11,8 & 32,3 & 55,9 & $6,48(<0,03)$ \\
\hline Peso & 18,5 & 18,5 & 63,0 & 35,3 & 22,1 & 42,6 & $3,54(<0,16)$ \\
\hline Altura & 3,7 & 7,4 & 88,9 & 17,6 & 26,5 & 55,9 & $9,31(<0,009)$ \\
\hline Geral & - & 11,1 & 88,9 & 7,4 & 39,7 & 52,9 & $10,94(<0,004)$ \\
\hline
\end{tabular}

$\mathrm{p}<0,05$

principal afazer, as atividades relacionadas à escola. Nenhum adolescente informou compromisso com atividade remunerada.

Em pesquisa realizada por Villas Boas ${ }^{22}$, envolvendo cerca de 130 jovens de ambos os sexos, da mesma região e nível socioeconômico, inferiu que $96 \%$ destes residiam na região do Grande ABC, com 66\% tendo seus estudos custeados por seus pais e $96 \%$ possuindo computador, sendo que a grande maioria $(86 \%) \mathrm{com}$ acesso à Internet em sua residência. Mesmo que estes jovens tenham participado das pesquisas em escolas distintas, preservam características comuns, como sendo da rede particular de ensino e mesma região, podendo-se estender os dados de Villas Boas ${ }^{22}$ para a presente pesquisa, no sentido de sua contextualização. Quanto aos adolescentes, pode-se inferir que pertencem a uma população de estrato socioeconômico elevado, com fácil acesso aos meios de comunicação como jornais, TV e Internet.

Em relação à insatisfação corporal, como uma expressão da forma como o adolescente se relaciona com seu corpo, esta parece ser uma caracterísitca peculiar ao jovem contemporâneo. Jovens de distintas nacionalidades revelam insatisfações focando o peso e áreas cor- porais $^{8,23,24}$.

Para o grupo de adolescentes estudados, também se evidenciou esta caracterísitica, com jovens expressando insatisfações corporais. As áreas de maior insatisfação referem-se ao estômago, coxas e ao peso corporal. Tendo como referência a fase de adolescência, meninos e meninas expressaram graus diferenciados de satisfação, com meninas pós-púberes mais insatisfeitas com inúmeras áreas corporais em relação às púberes. $\mathrm{O}$ mesmo não foi registrado entre os meninos, com somente uma área corporal com significância estatística.

O amadurecimento sexual, ou seja, a passagem do corpo infantil para o corpo adulto, repercutiu de forma distinta entre meninos e meninas. Para meninos, ocorreu uma preservação da forma de avaliação quanto à satisfação corporal, enquanto meninas diminuíram sensivelmente esta satisfação.

Áreas de insatisfação como busto, cintura, quadril, coxas e estômago, foram registradas igualmente por Davies e Furnham ${ }^{24}$ que constataram jovens pré-púberes mais satisfeitas quando comparadas às púberes. $\mathrm{O}$ mesmo observado por Willams e Currie ${ }^{8}$ (2000) que associaram fase de adolescência e imagem cor- 
poral, constatando meninas pós-púberes mais insatisfeitas em relação às pré-púberes.

Em estudo nacional, Vilela et al. ${ }^{25}$ registraram meninas mais insatisfeitas quando comparadas aos meninos, com $69 \%$ desejando perder peso. Archibald et al ${ }^{17}$, pesquisando meninas, constataram associação entre imagem corporal, desenvolvimento puberal e idade, com meninas pós-púbere mais insatisfeitas.

Os dados do presente estudo são consistentes com os reportados nos achados científicos inferindo que, com o desenvolvimento maturacional, meninas tendem a tornar-se mais insatisfeitas e meninos a preservar o critério de avaliação corporal.

A fase de adolescência mostrou ser um fator significativo para a compreensão da dinâmica feminina quanto à imagem corporal. $\mathrm{Na}$ tentativa de esclarecer este intrincado funcionamento, vários pesquisadores registraram importantes inter-relações.

Ricciardelli et al. ${ }^{24}$ constataram que as influências socioculturais afetam diferentemente meninos e meninas. Enquanto meninos são estimulados a praticarem atividades esportivas, meninas são estimuladas a praticarem atividades que impliquem em perda de peso. Observamos, assim, meninas recebendo reforço para cuidar-se, preocupando-se com o caráter estético e meninos focando o aspecto lúdico e relacional. Esta diferença no trato social possivelmente oferece aos jovens a aquisição de padrões estéticos e pessoais específicos, com as meninas tendendo a estar mais insatisfeita quando comparadas aos meninos ${ }^{8,27}$.

Siegel et al. ${ }^{10}$ complementam que a cultura contemporânea enfoca demasiadamente a magreza, propiciando às meninas um aumento crescente da insatisfação de acordo com seu crescimento. Brook e Tepper ${ }^{18}$ inferem que, com o desenvolvimento, meninas demonstram maior interesse por características corporais que se associam a atrativos sexuais, sugerindo que quanto mais atentas em relação às partes cor- porais que atraem ao sexo oposto, mais preocupadas e normalmente insatisfeitas estão com tais características. Conclui que o aumento da insatisfação para áreas como cintura e quadril pode relacionar-se à incorporação do ideal social de beleza e atração já internalizado.

Este padrão ideal de beleza pode ser observado já em crianças. Grogan e Wainwright $^{28}$ desenvolveram uma pesquisa qualitativa, constatando que as problemáticas envolvendo imagem corporal iniciam-se bem cedo, tão cedo quanto oito anos de idade, sugerindo a aceitação para o gênero feminino do padrão adulto de magreza.

Meninas possivelmente aumentam sua exigência para áreas corporais de acordo com o crescimento, em virtude da cobrança social de um padrão de corpo ideal, próximo à magreza. Meninos, em contraste, demonstram preferência por corpos grandes, aumentando a satisfação corporal de acordo com o desenvolvimento $^{10}$.

O modelo ideal de corpo propagado pela sociedade contemporânea exige para as meninas corpos magros, esbeltos e para meninos, corpo forte e musculoso. Segundo tal mensagem, aqueles que fogem a este ideal estão fadados a conviver com o fracasso e insatisfações. Esta insatisfação pode repercutir em como este adolescente se relaciona com seu corpo, no sentido de aceitá-lo e incorporá-lo como uma nova e definitiva entidade.

Pode-se inferir que a construção e elaboração do corpo pelo adolescente transcendem ao território do "privado". Sofre várias pressões, advindas de um território "coletivo", espaço social permeado pelas inter-relações. Nesta direção, o grupo de amigos e família, bem como as influências culturais, contribuem para esta construção, quer para aceitação total ou parcial ou até para a rejeição ${ }^{10,17}$.

Outro fator determinante refere-se à pressão da mídia televisiva e escrita, que atinge indiscriminadamente grande parte da po- 
pulação. Serra e Santos ${ }^{29}$ afirmam que o poder atual da mídia caracteriza-se como forma de produzir sentidos, projetando-os e legitimando-os, dando visibilidade aos fenômenos escolhidos por seus profissionais, no caso, os jornalistas.

A cultura contemporânea, por sua vez, com enfoque demasiado na magreza e por meio da pressão da mídia, transforma o corpo em um objeto de manipulação e projeção de desejos, partindo-se do modelo vigente de padrão feminino de beleza. Para meninos, em contraponto, os apelos induzem ao tamanho e força corporal $^{20}$.

Attie e Brooks-Gunn ${ }^{31}$ constataram a insatisfação corporal como único fator significante entre outros estudados, que prognosticou o desenvolvimento de futuros problemas alimentares. No Brasil pouco se tem feito sobre o tema. Piccinini et al..$^{32}$, pesquisando os estereótipos sociais vinculados aos formatos corporais, constataram o quanto jovens são discriminados e excluídos quando fogem a um padrão ideal de apresentação corporal exigido implicitamente nas relações sociais.

Desta forma, meninas em fase de crescimento, mostraram-se mais suscetíveis ao desencadeamento da insatisfação corporal. Parece que ser criança implica em uma vivência de maior satisfação corporal. $\mathrm{O}$ amadurecimento sexual, com o ganho do corpo adulto, traz em si, possivelmente, uma carga de cobranças, o que implicaria na obtenção de um suposto corpo tido como ideal, muitas vezes, quase inatingível. Para os meninos, não se observou este efeito, tendendo a preservar a satisfação, demonstrando assim maior aceitação corporal.

A literatura tem indicado que a idade é um importante fator, sendo necessário controlá-lo nas pesquisas envolvendo imagem corpo$\mathrm{ral}^{11,12}$. Sugere-se mais estudos nesta linha de pesquisa, visto a escassez de registros nacionais que envolvam a população e o tema pesquisado.

Sabe-se ainda que o fenômeno "imagem corporal" não é totalmente compreendido, sendo necessário aproximá-lo de diferentes aspectos, o que incluiria o psicológico, psiquiátrico, somático, neurológico entre outros ${ }^{11}$.

\section{CONCLUSÃO}

Meninas pós-púberes expressaram maior insatisfação quando comparadas às pré-púberes, sendo que o mesmo não ocorreu com os meninos, com o grupo dos pré-púberes reportando somente uma área de insatisfação.

Conclui-se que meninas pós-púberes mostraram-se mais suscetíveis ao desencadeamento da insatisfação corporal quando comparada aos seus pares.

\begin{abstract}
Severe behavioral problems related to feeding habits, with repercussion on body presentation, perception and image, and related to feeding practices have, nowadays, its prevalence increased. The present study aimed to verify adolescents' perception regarding body image dissatisfaction. We analyzed students of a private school, aged 10 to 14 , of both sexes. The boys and girls were divided for the analysis based on their age, transformed into sexual maturation phase. To assess body image perception, an adapted body satisfaction scale was applied, composed of 15 body areas. Boys showed more dissatisfaction with weight, waist and stomach. Girls with weight, chest/breast and stomach. The association between sexual maturation and body satisfaction was significant, among the boys, for the shoulder/back area, while among girls, the significant areas were: face, hair, hip, stomach, waist, chest/breast, muscle strength, height and general aspects. We concluded that post-pubescent girls proved to be more susceptible to the manifestation of body dissatisfaction when compared with their peers.
\end{abstract}

Key words: Adolescence. Body image. Sexual maturation. 


\section{REFERÊNCIAS}

1. Osório LC. Adolescente hoje. $3^{\mathrm{a}}$ ed. Porto Alegre: Artes Médicas; 1992.

2. [WHO] World Health Organization. Physical sta tus: use and interpretation of anthropometry. Gen ova; 1995.

3. Schoen-Ferreira TH, Aznar-Farias M, Silvares EFM. A construção da identidade em adolescente: um estudo exploratório. Est. Psicologia. 2003; 8(1): 107-15.

4. Ozella S. Adolescências construídas. A visão da psicologia sócio-historica. São Paulo: Cortez Ed itora; 2003.

5. Salles LMF. Adolescência, escola e cotidiano. Contradições entre o genérico e o popular. Pirac icaba: Editora Unimep; 1998.

6. Vaccari VL. Saúde sexual, escolas promotoras de saúde e relações de gênero: representações de adolescentes sobre masculinidades e feminil idades [dissertação]. São Paulo (SP): Faculdade de Saúde Publica da Universidade de São Paulo; 2002.

7. Gambardella AMD. Práticas alimentares de ado lescentes. Rev Nutr. 1999;1: 55-9.

8. Willams J.M, Currie C. Self-esteem and physical development in early adolescence: pubertal time and body image. J Early Adolesc. 2000; 20(2): 12949.

9. [WHO] World Health Organization. What about boys? A literature review on the health and de velopment of boys adolescents. Switzerland; 2000.

10. Siegel JM, Yancey AK, Aneshensel CS, Schler R. Body image, perceived pubertal timing and ado lescent mental health. J Adolesc Health. 1999; 25 : 155-65.

11. Brodie DA, Bagley K, Slade PD. Body image per ception in pre-and post adolescent females. Per cep Mot Skills. 1994; 78:147-54.

12. O’Dea JÁ, Caputi P. Association between socio economic status, weight, age and gender, and the body image and weight control practices of 6- to 19- year-old children and adolescent. Health Educ Res. 2001; 16(5): 521-32.

13. Richards MH, Petersen AC, Boxer AM, Albrecht $\mathrm{R}$. Relation of weight to body image in pubertal girls and boys from two communities. Dev Psy chol. 1990; 26(2): 313-21.

14. Fleitlich B.W. O papel da imagem corporal e os riscos de transtornos alimentares. Pediatria Mod erna 1997; 32(1/2): 56-62.
15. Atkinson RS, Nitzke AS. School based programs on obesity. BMJ. 2001; 323(7320): 1018-9.

16. Wang, T, Monteiro C, Popkin BM. Trends of obe sity and underweight in older children and ado lescent in the United States, Brazil, China and Russia. Am J Clin Nutr. 2002; 75: 971-7.

17. Archibald, AB, Graber JA, Brooks-Gunn J. Asso ciation among parent-adolescent relationship, pu bertal growth, dieting, and body image in young adolescent girls: A short-term longitudinal study. J Res Adolesc. 1999; 9(4): 395-415.

18. Brook, U, Tepper I. High school students' atti tudes and knowledge of food consumption and body image: Implications for school based edu cation. Patient Educ Couns. 1997; 30: 283-8.

19. Guedes MLS, Guedes IS. Bioestatística para profissionais de saúde. $1^{\mathrm{a}}$ ed. Rio de Janeiro, Brasília: ao Livro Técnico/CNPq; 1988.

20. Brown, TA, Cash TF, Mikulka PJ. Attitudinal body-image assessment: Factor analysis of the body-self relations questionnaire. J Pers Assess. 1990; 55: 135-44.

21. Loland, NW. Body image and physical activity. A survey among Norwegian men and women. Int J Sport and Psychol. 1998; 29: 339-65.

22. Villas-Boas JPC. Adolescentes em situação de pré-vestibular: atividade física e estresse [disser tação]. São Paulo (SP): Faculdade de Saúde Publi ca da Universidade de São Paulo; 2002.

23. Cuadrado C, Carbajal A, Moreiras O. Body per ceptions and slimming attitudes reported by Span ish adolescents. Eur J Clin Nutri. 2000; 54(1): 658.24. Davies E, Furnham M. Body satisfaction in adolescent girls. Brit J Med Psychol. 1986; 59: 279-87.

25. Vilela JEM, Lamounier JA, Dellaretti Filho MA, Neto JRB, Horta GM. Transtornos alimentares em escolares. J Pediatr. 2004; 80: 49-54.

26. Ricciardelli, LA, McCabe MP, Banfield S. Body image and body change methods in adolescent boys role of parents, friends and the media. $\mathbf{J}$ Psych Res. 2000; 49: 189-97.

27. Feingold A, Mazella R. Gender differences in body image are increasing. Psycol Science. 1998; 9(3): 190-5.

28. Grogan S, Wainwright T. Growing up in the cul ture of slenderness - Girl's experience of body dissatisfaction. Women's Stud Int Forum. 1996; 19(6): 665-73.

29. Serra GMA, Santos EM. Saúde e mídia na con strução da obesidade e do corpo perfeito. Ciênc Saúde Coletiva. 2003; 8(3): 691-701.

30. Bergstrom E, Stenlund H, Svedjehall B. Assess 
ment of body perception among swedish adoles cent and young adults. J Adolesc Health. 2000; 26: $70-5$.

31. Attie I, Brooks-Gunn J. Development of eating problems in adolescent girls: A longitudinal study.
Dev Psychol. 1989; 189 (25): 70-9.

32. Piccinini CA, Benincá C, Hennigen I, Hernandez JAE. Estereótipos sociais vinculados aos forma tos corporais em adolescentes. Aletheia. 1996; 3 : 11-6.

Recebido em 22/02/2005

Modificado em 25/03/2005

Aprovado em 05/04/2005 